УДК 512.546

Е. Г. Зеленюк (Луцк. инлустр. ии-т)

\title{
РАЗЛОЖИМОСТЬ ТОПОЛОГИЧЕСКИХ ГРУПП
}

We prove that every countable Abelian group with a finite number of second order elements can be decomposed into countable number of subsets which are dense in any nondiscrete group topology.

Доведено, що кожну зчислепиу абелеву групу з скінченним числом елементів порядку 2 можна разбити на зчисление число пі/миожин шільних у будь-якій неднскретній груповія топології.

Введение. Топологическая группа называется неразложимой ( $\boldsymbol{\kappa}_{0}$-неразложимой), если ее нельзя разбить на два (на $\aleph_{0}$ ) плотных подмножества. В работе [1] В. В. Комфорт и Я. ван Милл доказали, что недискретная неразложимая топологическая абелева группа содержит бесконечную булеву подгруппу. Булевой называется группа периода 2. Эквивалентная формулировка теоремы Комфорта - Милла: недискретная топологическая абелева группа с конечным числом элементов порядка 2 разложима. Ранее В. И. Малыхин в предположении аксиомы Мартина построил на счетной булевой группе групповую топологию с единственным свободным сходящимся к нулю ультрафильтром [2]. Группа Малыхина неразложима. Следовательно, каждая абелева группа, содержащая бесконечную булеву подгруппу, в предположении аксипмы Мартина допускает недискретную неразложимую групповую топологию. „Наивные” примеры недискретных неразложимых топологических групп неизвестны. Это одна из проблем, поставленных в [1]. Далее теорема Комфорта - Милла была усилена И. В. Протасовым следующим образом: недискретная неразложимая топологическая абелева группа содержит открытую счетную булеву подгруппу [3]. Недискретная топологическая абелева группа с конечным числом элементов порядка $2 \aleph_{0}$-разложима [4].

Группа называется абсолютно разложимойі (абсолютно $\boldsymbol{\aleph}_{0}$-разложимой), если се можно разбить на два (на $\aleph_{0}$ ) подмножества, плотные в любой недискретной групповой топологии. Проблема опнсания абсолютно разложимых групп была поставлена в [1]. И. В. Протасов, используя различные методы, доказал абсолютную разложимость и абсолютную $\aleph_{0}$-разложимость многих групп. Один из наиболее тонких результатов в этом направлении - абсолютная разложнмость группы рациональных чисел $\mathbb{Q}$ [5]. Однако уже вопросы об абсолютной разложимости $\mathbb{Q} \oplus \mathbb{Q}$ и абсолютной $\aleph_{0}$-разложимости $\mathbb{Q}$ оставались открытыми.

Основныс результаты данной работы составляют две теоремы, в которых установлено, что в классе абелевых и в классе счетных периодических групп каждая недискретная $\aleph_{0}$-неразложимая группа содержит открытую счетную булеву подгруппу и каждая счетная абелева группа с конечным числом элементов порядка 2 абсолютно $\aleph_{0}$-разложима. Обе теоремы доказываются единым методом, берущим начало с работы [6]. Этому методу посвящен первый пункт. Во втором пункте с его помощью доказываются основные теоремы. Все топологии предполагаются хаусдорфовыми.

1. Локалыные левотопологические группы и их автоморфизмы. Левотопологической группой называется группа, снабженная топологией, в которой непрерывны левые сдвиги. Топологическое пространство $X$ с выделенным элементом $e$ (единицей) и частнчной бинарной операциеи (умножением) называется локальной левотопологической группой, если существует левотопологическая группа $G$ такая, что 1) $e$-единица $G, 2) X$ - открытая окрестность $e \in G, 3$ ) частичное умножение на $X$ - это в точности частичная операция, индуцированная на $X$ умножением на $G$. 
Локальная левотопологическая группа $X$ называется регулярной (счетного характера), если пространство $X$ регулярно (имеет счетную базу окрестностей единицы). Регулярность пространства означает наличие в каждой точке базы из замкнутых окрестностей. Для счетных пространств регулярность эквивалентна нульмерности - наличию в каждой точке базы из открыто-замкнутых окрестностей. Существуют нерегулярные левотопологические группы.

Пример. Пусть $(G, \tau)$ - левотопологическая группа, содержащая последовательность $\left\{a_{n}: n<\omega\right\}$ неединичных элементов, сходящихся к единице, $\tau^{\prime}$ - топология на $G$, базу которой образуют множества вида $x\left(U \backslash\left\{a_{n}: n<\right.\right.$ $\omega\}$ ), где $x \in G, U$ - открытая окрестность единицы $(G, \tau)$. Тогда $\left(G, \tau^{\prime}\right)-$ левотопологическая группа, любая замкнутая окрестность единицы которой содержит почти всю последовательность $\left\{a_{n}: n<\omega\right\}$. Следовательно, $\left(G, \tau^{\prime}\right)$ не регулярна.

Пусть $X, Y$ - локальные левотопологические группы. Отображение $f: X \rightarrow$ $\rightarrow Y$ называется гомоморфизмом, если для любого $x \in X$ найдется окрестность $U$ единицы $e_{X} \in X$ такая, что для всех $z \in U$ произведения $x z, f(x) f(z)$ определены и $f(x z)=f(x) f(z)$. Если $f: X \rightarrow Y-$ гомоморфизм, то $f\left(e_{X}\right)=e_{Y}$. Инъективный гомоморфизм называется изоморфизмом. Топологический изоморфизм локальной левотопологической группы на себя называется автоморфизмом. Порядком автоморфизма $f: X \rightarrow Y$ называется inf $\sup \{|O(x)|: x \in$ $\in U\}$, где $O(x)$ - орбита $x$ относительно $f$, а inf берется по всевозможным окрестностям единицы $U$. Автоморфизм порядка больше 1 называется нетривиальным. Автоморфизм называется однородным, если орбита всех неединичных элементов равномощны.

Лемма 1. Пусть $G$ - недискретнал топологическал группа без открытых булевьх подгрупп. Тогда на $G$ существует нетривиальный автольорфизл. Если $G$ абелева либо периодическал, то на $G$ существует нетривиальный автомюрфизм конечного порядка.

Доказательство. Для каждого элемента $a \in G$ рассмотрим автоморфизм $x \mapsto a^{-1} x a$. Предположим, что каждый такой автоморфизм тривиален. Это означает, что для любого элемента $a \in G$ найдется окрестность единицы $U$ такая, что $a z=z a$ для всех $z \in U$. Покажем, что отображение $x \mapsto x^{-1}$ будет автоморфизмом. Пусть $x$ - произвольный элемент из $G, U$ - окрестность единицы такая, что $x z=z x$ для всех $z \in U$, тогда $(x z)^{-1}=z^{-1} x^{-1}=x^{-1} z^{-1}$. Поскольку $G$ без открытых булевых подгрупп, то этот автоморфизм нетривиален.

Лемма 2. Пусть Х-недискретнал локальная левотопологическал группа, $f$-автохьорфизм на $X$ конечного порлдка т. Существует недискретная локальная левотопологическал группа $X_{0}$, однородный автоморфизм $f_{0}$ на $X_{0}$ порлдка $m$ и непрерывный автомьорфизя $h: X_{0} \rightarrow X$ такие, что $h \cdot f_{0}=f \cdot h$. Причель, если $X$ регулярна, то $X_{0}$ также жожно выбрать регулярной.

Доказательстөо. Выделяя в $X$ достаточно малую открытую окрестность единицы, можно считать , что для любого $x \in X,|O(x)| \leq m$. Рассмотрим множество $M=\{x \in X:|O(x)|=m\}$. Оно открыто. Следовательно, для любого $x \in M$ наидется окрестность единицы $U$ такая, что $x U \subseteq M$. Кроме этого, $e \in \bar{M}$. Пусть $X_{0}=\{e\} \cup M, h: X_{0} \rightarrow X-$ естественное вложение, 
$f_{0}=\left.f\right|_{X_{0}}$. Снабдим $X_{0}$ топологией, объявив окрестностями точки $x \in X_{0}$ множества вида $x\left(U \cap X_{0}\right)$, где $U$ - достаточно малая окрестность $e \in X$. Тогда $X_{0}$ - недискретная локальная левотопологическая группа, причем, если $X$ регулярна, то регулярна и $X_{0}, h: X_{0} \rightarrow X$ - непрерывнын изоморфизм, $f_{0}: X_{0} \rightarrow X_{0}$ - однородный автоморфизм порядка $m, h \cdot f_{0}=f \cdot h$.

Пусть $\mathbb{Z}(m+1)=\{0,1, \ldots, m\}$ - џнклическая группа порядка $m, g$ - подстановка на $\mathbb{Z}(m+1)$, заданная циклом $(1, \ldots, m), \underset{⿴ 囗 十}{\oplus}(m+1)$ 一 прямая сумма $\omega$ экземпляров $\mathbb{Z}(m+1)$, снабженная обычной топологией суммы $l, r-$ отображения, сопоставляющие каждому ненулевому элементу из $\underset{\emptyset}{\mathbb{Z}}(m+1)$ номер первой и последней ненулевой координаты. Подстановка $g$ на $\mathbb{Z}(m+1)$

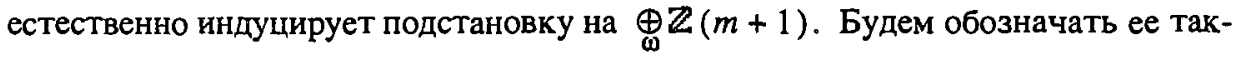
же $g$. Очевидно, что $g$ - гомеоморфизм, $g(0)=0$ орбиты всех ненулевых

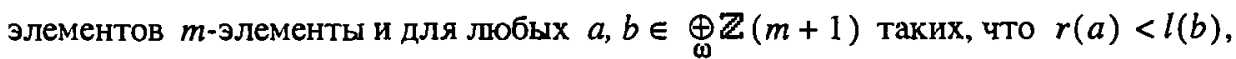
имеем $g(a+b)=g(a)+g(b)$. Следовательно, $g$ - однородныи автоморфизм порядка $m$ на локальной левотопологической группе $\underset{\omega}{\oplus} \mathbb{Z}(m++1)$.

Теорема 1. Пусть X-счетная недискретная регулярная локальнаялевотопологическая группа, $f$-однородный автольрфизль на $X$ порядка $\mathrm{m}$.

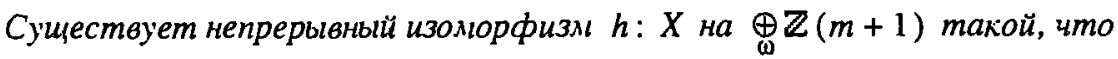

1) если $x, y \in X u r(h(x))+1<l(h(y))$, то произведение $x y$ определено $u h(x y)=h(x)+h(y)$,

2) $h \cdot f=g \cdot h$.

Если $X$ счетного характера, по непрерывный изомюрфизл $h$ мохно сделать mononozllyeckllst.

Доказательству теоремы 1 предпошлем следующую лемму.

Лемма 3. Пусть $X$ - счетное регулярное пространство, $f$ - гомеольрфизм на $X$, относительно которого каждый элемент из $X$ илеет т-элежентную орбиту. Тогда существует разбиение $X$ на открыто-залинутые льножества $A_{1}, \ldots, A_{m}$ такие, ито $f\left(A_{1}\right)=A_{2}, f\left(A_{2}\right)=A_{3}, \ldots, f^{m-1}\left(A_{m}\right)=A_{1}$.

Доказапельспво. Занумеруем элементы множества $X$ натуральными числами: $X=\left\{x_{n}: n<\omega\right\}$. Рассмотрим орбиту $\left\{x_{0}, f\left(x_{0}\right), \ldots, f^{m-1}\left(x_{0}\right)\right\}$ элемента $x_{0}$. Выберем открыто-замкнутую окрестность $U_{0}$ элемента $x_{0}$ такую, что множества $U_{0}, f\left(U_{0}\right), \ldots, f^{m-1}\left(U_{0}\right)$ дизъюнктны, и положим

$$
A_{1}^{0}=U_{0}, \quad A_{2}^{0}=f\left(U_{0}\right), \ldots, A_{m}^{0}=f^{m-1}\left(U_{0}\right), \quad X_{0}=A_{1}^{0} \cup \ldots \cup A_{m}^{0} .
$$

Далее, в последовательности $\left\{x_{n}: n<\omega\right\}$ ищем первый элемент, который не принадлежит множеству $X_{0}$. Не умаляя общности, пусть это будет $x_{1}$. Выбираем открыто-замкнутую окрестность $U_{1} \subset X \backslash X_{0}$ элемента $x_{1}$ такую, что множества $U_{1}, f\left(U_{1}\right), \ldots, f^{m-1}\left(U_{1}\right)$ дизъюнктны, и полагаем

$$
\begin{gathered}
A_{1}^{1}=A_{1}^{0} \cup U_{1}, \quad A_{2}^{1}=A_{2}^{0} \cup f\left(U_{1}\right), \ldots, A_{m}^{1}=A_{m}^{0} \cup f^{m-1}\left(U_{1}\right), \\
X_{1}=A_{1}^{1} \cup \ldots \cup A_{m}^{1} .
\end{gathered}
$$

Продолжая таким образом, построим возрастающие последовательности 
множеств $\left\{A_{1}^{n}: n<\omega\right\}, \ldots,\left\{A_{m}^{n}: n<\omega\right\}$, объединения которых $A_{1}=$ $=\bigcup\left\{A_{1}^{n}: n<\omega\right\}, \ldots, A_{m}=\bigcup\left\{A_{m}^{n}: n<\omega\right\}$ и будут требуемыми множествами.

Доказательство теоремы 1. Пусть $F$ - полугруппа слов в алфавите $\mathbb{Z}(m+1)$ с пустым словом $\varepsilon, L_{n}-$ множество всех слов из $F$ длины $n, S_{j}^{n}-$ множество всех слов из $L_{n}$, в которых первые $j$ букв нулевые, а остальные ненулевые,

$$
0 \leq j \leq n, \quad S_{n}^{n}=\left\{\theta_{n}\right\}, \quad S_{n}=\bigcup\left\{S_{j}^{n}: j \leq n\right\}, \quad S=\bigcup\left\{S_{n}: n<\omega\right\} .
$$

Подстановка $g$ на $\mathbb{Z}(m+1)$ естественно индуцирует подстановку на $F$. Будем обозначать ее также $g$. Очевидно, что все множества $S_{j}^{n}, S_{n}, L_{n}, S$ инвариантны относительно $g$ и орбиты всех непустых слов из $F$, отличных от $\theta_{n}, m-$ элементы. Пусть $s-$ произвольное слово из $F$. Если $s \in S$, то положим $s^{\prime}=\varepsilon, s^{*}=s$. Если же $s \in F \backslash S$, то $s$ однозначно раскладывается в произведение $s_{1} \ldots s_{k+1}$, где

$$
\begin{gathered}
s_{1} \in S_{j_{1}}^{n_{1}}, \ldots, s_{k+1} \in S_{j_{k+1}}^{n_{k+1}}, \quad 0 \leq j_{1}<n_{1}, \quad 0<j_{2}<n_{2}, \ldots, \\
0<j_{k}<n_{k}, \quad 0<j_{k+1} \leq n_{k+1} .
\end{gathered}
$$

В этом случае положим $s^{\prime}=s_{1} \ldots s_{k}, s^{*}=\theta_{n_{1}+\ldots+n_{k}} s_{k+1}$.

Пусть $X=\left\{e, x_{1}, x_{2}, \ldots\right\}$. Каждому слову $s \in F$ сопоставим непустое открыто-замкнутое подмножество $X(s) \subseteq X$ и точку $x(s) \in X(s)$ такие, что $X(\varepsilon)=X, x(\varepsilon)=e$ и для каждого $n \geq 1$ выполняются следующие условия:

1) ${ }_{n}$ для каждого $s \in S_{n+1}$ множества $X(s-1), i \leq m$, образуют разбиение $X(s), x(s 0)=x(s)$,

2), для всех $s \in S_{n-1}, y \in X\left(\theta_{n}\right)$ произведения $x(s) y$ определены н $f(x(s) y)=f(x(s)) f(y)$,

3) для каждого $s \in S_{n} f(X(s))=X(g(s)), f(x(s))=x(g(s))$,

4) $)_{n}$ для каждого $s \in L_{n} X(s)=x\left(s^{\prime}\right) X\left(s^{*}\right), x(s)=x\left(s^{\prime}\right) x\left(s^{*}\right)$,

5) $x_{n} \in\left\{x(s) s \in L_{n}\right\}$.

Выберем открыто-замкнутую инвариантную (относительно $f$ ) окрестность единицы $U_{1}$ такую, что $x_{1} \notin U_{1}$. Тогда множество $X \backslash U_{1}$ также открытозамкнуто и инвариантно. Согласно лемме 3 его можно разбить на открытозамкнутые подмножества $A_{1}, \ldots, A_{m}$ такие, что $f\left(A_{1}\right)=A_{1}, f\left(A_{2}\right)=A_{3}, \ldots$, $f\left(A_{m}\right)=A_{1}$. Выберем элемент $a \in A_{1}$ такой, что $x_{1} \in O(a)$. Положим

$$
\begin{gathered}
X(0)=U_{1}, \quad X(1)=A_{1}, \quad X(2)=A_{2}, \ldots, X(m)=A_{m}, \\
x(0)=e, \quad X(1)=a, \quad X(2)=f(a), \ldots, x(m)=f^{m-1}(a) .
\end{gathered}
$$

Фиксируем $n \geq 1$ и предположим, что уже определены $X(s), x(s)$ для всех $s \in \bigcup\left\{L_{j}: j \leq n\right\}$, прнчем выполнякотся условия $1_{j}-5_{j}$ для всех $j \leq n$. Отределим $X(s), x(s)$ для $s \in L_{n+1}$.

Покажем, что в условиях $1_{n}-5_{n}$ можно $S$ заменить на $L$ :

1) $x(s 0)=x\left((s 0)^{\prime}\right) x\left((s 0)^{*}\right)=x(\tilde{s}) x\left(\theta_{n}\right)=x(s) e=x(s)$ ( $\tilde{s}-$ слово, полученное из $s$ удаленнем нулевого хвоста), 


$$
\begin{gathered}
X(s 0)=x\left((s 0)^{\prime}\right) X\left((s 0)^{*}\right)=x(s) X\left(\theta_{n}\right)=x\left(s^{\prime}\right) x\left(s^{*}\right) X\left(\theta_{n}\right)=x\left(s^{\prime}\right) X\left(s^{*} 0\right), \\
X(s i)=x\left((s i)^{\prime}\right) X\left((s i)^{*}\right)=x\left(s^{\prime}\right) X\left(s^{*} i\right), \quad 1 \leq i \leq m ; \\
\text { 2) } f(x(s) y)=f\left(x\left(s^{\prime}\right) x\left(s^{*}\right) y\right)=f\left(x\left(s^{\prime}\right)\right) f\left(x\left(s^{*}\right) y\right)=f\left(x\left(s^{\prime}\right)\right) f\left(x\left(s^{*}\right)\right) f(y)= \\
=f\left(x\left(s^{\prime}\right) x\left(s^{*}\right)\right) f(y)=f(x(s)) f(y) ; \\
\text { 3) } f(x(s))=f\left(x\left(s^{\prime}\right) x\left(s^{*}\right)\right)=f\left(x\left(s^{\prime}\right)\right) f\left(x\left(s^{*}\right)\right)=x\left(g\left(s^{\prime}\right)\right) x\left(g\left(s^{*}\right)\right)=x(g(s)) .
\end{gathered}
$$

Рассмотрим разбиение множества $X$ множествами $X(s), s \in L_{n}$. Одно из них, скажем $X\left(s_{0}\right)$, содержит $x_{n+1}$. Поскольку $X\left(s_{0}\right)=x\left(s_{0}^{\prime}\right) X\left(s_{0}^{*}\right)$, то существует $y_{n+1} \in X\left(s_{0}^{*}\right)$ такой, что $x_{n+1}=x\left(s_{0}^{\prime}\right) y_{n+1}$. Выберем открыто-замкнутую инвариантную окрестность единищы $U_{n+1}$ такую, что для всех $s \in S_{n}$, $y \in U_{n+1}$ произведения $x(s) y$ определены, $f(x(s) y)=f(x(s)) f(y), x(s) U_{n+1} \subset$ $\subset X(s)$ и $y_{n+1} \notin x\left(s_{0}^{*}\right) U_{n+1}$, если $y_{n+1} \neq x\left(s_{0}^{*}\right)$.

Разберемся вначале с множеством $X\left(\theta_{n}\right)$. Разобьем множество $X\left(\theta_{n}\right) \backslash U_{n+1}$ на открыто-замкнутые подмножества $B_{1}, \ldots, B_{m}$ такие, что $f\left(B_{1}\right)=B_{2}$, $f\left(B_{2}\right)=B_{3}, \ldots, f\left(B_{m}\right)=B_{1}$ (лемма 3). Выберем элемент $b \in B_{1}$ такой, что $y_{n+1} \in O(b)$, если $s_{0}^{*}=\theta_{n}$. Положим

$$
\begin{aligned}
& X\left(\theta_{n} 0\right)=U_{n+1}, \quad X\left(\theta_{n} 1\right)=B_{1}, \quad X\left(\theta_{n} 2\right)=B_{2}, \ldots, X\left(\theta_{n} m\right)=B_{m}, \\
& x\left(\theta_{n} 0\right)=e, \quad x\left(\theta_{n} 1\right)=b, \quad x\left(\theta_{n} 2\right)=f(b), \ldots, x\left(\theta_{n} m\right)=f^{m-1}(b) .
\end{aligned}
$$

Пусть теперь $p$ - произвольное слово из $S_{n}$, отлюгное от $\theta_{n}, O(p)-$ его орбита. Разобьем множество $X(p) \backslash x(p) U_{n+1}$ произвольно на открыто-замкнутые подмножества $C_{1}, \ldots, C_{m}$ и выберем элементы $c_{1} \in C_{1}, \ldots, c_{m} \in C_{m}$ такие, что $y_{n+1} \in O\left(c_{1}\right) \cup \ldots \cup O\left(c_{m}\right)$, если $y_{n+1} \in \bigcup\left\{O(x): x \in X(p) \backslash x(p) U_{n+1}\right\}$. Положим

$$
\begin{gathered}
X(p 0)=x(p) U_{n+1}, \quad X(p 1)=C_{1}, \ldots, X(p m)=C_{m}, \\
x(p 0)=x(p), \quad x(p 1)=c_{1}, \ldots, x(p m)=c_{m} .
\end{gathered}
$$

Для $s \in O(p) \backslash\{p\} X(s), x(s)$ огределим условием $3_{n+1}$.

После того, как $X(s), x(s)$ определены для всех $s \in S_{n+1}$, определим $X(s)$, $x(s)$ для всех $s \in L_{n+1} \backslash S_{n+1}$ условием $4_{n+1}$. Заметим, что если $x_{n+1} \notin$ $\notin\left\{x(s): s \in L_{n}\right\}$, то $x_{n+1}=x\left(s_{0}^{\prime}\right) y_{n+1}=x\left(s_{0}^{\prime}\right) x\left(s_{0}^{*} i\right)=x\left(s_{0} i\right)$ для некоторого $i \neq 0$.

Из условия $5_{n}$ следует, что построенное отображение $F \ni s \mapsto x(s) \in X$

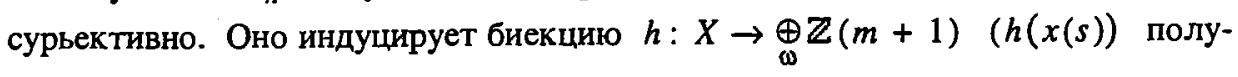
чается из $s$ приписыванием нулевого хвоста).

Поскольку множества $X(s), X(s 0), X(s 00), \ldots$ - окрестности точки $x(s)$, то биекция $h$ непрерывна. Условия 1,2 следуют из $4_{n}, 3_{n}$, в частности, $h-$ изоморфизм. Если $X$ счетного характера, то последовательность $X\left(\theta_{1}\right)$, $X\left(\theta_{2}\right), \ldots$ можно сделать базой окрестностей единицы и тогда $h$ будет гомеоморфизмом.

2. Основные результаты. Топологическое пространство называется разложимым ( $\kappa_{0}$-разложимым), если его можно разбитъ на два (на $N_{0}$ ) плотных подмножества. Отметим следуюшие простые утверждения о разложимости. 
1. Непрерывный инъективный образ разложимого ( $\aleph_{0}$-разложимого) пространства разложим ( $\boldsymbol{K}_{0}$-разлюжнм).

2. Замыкание разложимого ( $\boldsymbol{\kappa}_{0}$-разложимого) подпространства разложимо ( $\aleph_{0}$-разложимо).

3. Если однородное пространство содержит разложимое $\left(\boldsymbol{K}_{0}\right.$-разложимое) подпространство, то оно и само разложимо ( $\aleph_{0}$-разложимо).

Первые два утверждения очевидны, третъе легко следует из леммы Куратовского - Цорна, утверждения 2 и однородности.

Теорема 2. Пусть $(X, \tau)-$ счетная недискретная регулярная локальная левотопологическая группа, $f$-нетривиальный однородный автояюрфизя на $(X, \tau)$ конечного порядка. Тогда существует разбиение множества $X$ на счетное число подножеств, плотны в любой недискретной топологии $\tau^{\prime}$ на $X$ такой, что

1) $\left(X, \tau^{\prime}\right)$ - локальнал левотопологическал группа,

2) $f$ - непрерывное отображение в топологии $\tau^{\prime}$,

3) каждая окрестность единццы в топологии $\tau^{\prime}$ нетривиально пересекает каждую окрестность единицы в топологии $\tau$.

Доказательство. Пусть $m$ - порядок автоморфизма $f, h: X \rightarrow \underset{\omega}{\mathbb{Z}}(m+$ +1 ) 一 непрерывный изоморфизм, предоставляемый теоремой 1 . Для каждого элемента $x \in X$ через $\delta(x)$ обозначим количество пар соседних элементов в последовательности ненулевых координат $h(x)$, отличных от пар $(a, a)$, где $a \in\{1, \ldots, m\}$.

Заметим что для любого $x \in X \delta(x)=\delta(f(x))$ и для любых $x, y \in X$ таких, чाо $r(h(x))+1<l(h(y))$,

$$
\delta(x y)= \begin{cases}\delta(x)+\delta(y), & \text { если } \rho(x)=\lambda_{1}(y), \\ \delta(x)+\delta(y)+1, & \text { если } \rho(x) \neq \lambda(y),\end{cases}
$$

где $\lambda(x), \rho(x)$ - первая и последняя ненулевые координаты $h(x)$. Следовательно, для любых $x, y \in X$ таких, что $r(h(x))+1<l(h(y))$, существует $i<m$ такое, что

$$
\delta\left(x f^{i}(y)\right)=\delta(x)+\delta(y), \quad \delta\left(x f^{i+1}(y)\right)=\delta(x)+\delta(y)+1,
$$

Положим

$$
X_{n}=\left\{x \in X: \delta(x) \neq 0, \delta(x) \equiv 2^{n}\left(\bmod 2^{n+1}\right)\right\} .
$$

Иными словами, $X_{n}$ - множество всех таких $x \in X$, что $\delta(x) \neq 0$ н разложение числа $\delta(x)$ на простые множители содержит $n$ 2-ек. Очевидно, что множества $X_{n}$ образуют разбиение множества $X \backslash\{x \in X, \delta(x)=0\}$. Покажем, что каждое $X_{n}$ плотно в $\left(X, \tau^{\prime}\right)$. Рассмотрим произвольный элемент $x \in X$ и окрестность единицы $U$ в топологии $\tau^{\prime}$. Пложим $k=2^{n+1}-1$ и выберем в $U$ элементы $x_{1}, \ldots, x_{k}$ такие, что

1) $r(h(x))+1<l\left(h\left(x_{1}\right)\right), r\left(h\left(x_{j}\right)\right)+1<l\left(h\left(x_{j+1}\right)\right)$,

2) $y_{1}, \ldots, y_{k} \in U$ для любых $y_{j} \in O\left(x_{j}\right)=\left\{x_{j}, f\left(x_{j}\right), \ldots, f^{m-1}\left(x_{j}\right)\right\}$.

Тогда среди элементов $x y_{1} \ldots x y_{k} \in x U$, где $y_{j} \in O\left(x_{j}\right)$, обязательно имеется элемент из $X_{n}$.

Из леммы 2 и теоремы 2 вытекает следующее утверждение. 
Следствие 1. Если на счетной недискретной регулярной локальной левотопологической группе существует нетривиальный автоморфизм конечного порядка, то она $\aleph_{0}$-разложияла.

Дисперсионным характером точки в топологическом пространстве называется наименьшая из мощностей баз ее окрестностей.

И. В. Протасов доказал, что каждую несчетную абелеву группу можно разбить на счетное число подмножеств, плотных в любой недискретной групповой топологии несчетного дисперсионного характера. Следовательно, каждая $\boldsymbol{\kappa}_{0-}$ неразложимая абелева группа имеет счетный дисперсионный характер [4]. Отсюда, из леммы 1 и из следствия 1 вытекает справедливость такого утверждения.

Cледствие 2. В классе абелевых и в классе счетных периодических групп каждая недискретная $\aleph_{0}$-неразложильая группа содержит открытую счетную булеву nодгрупnу.

Bonpoc 1. Верно ли, что каждую несчетную группу можно разбить на счетное число подмножеств, плотных в любой недискретной групповой топологии несчетного дисперсионного характера ?

Bonpoc 2. Верно ли, что каждая счетная недискретная $\aleph_{0}$-неразложимая группа содержит открытую булеву подгруппу ?

Группа называется абсолютно разложимой (абсолютно $\boldsymbol{\kappa}_{0}$-разложимой), если ее можно разбить на два (на $\aleph_{0}$ ) подмножества, плотные в любой недискретной групповой топологии.

Следствие 3. Каждая счетная абелева группа с конечным числом элементов порлдка 2 абсолютно К $\aleph_{0}$-разложильа.

Доказательство. Пусть $G$ - произвольная счетная абелева группа с конечным числом элементов порядка 2. Снабдим ее какой-то групповой предкомпактной топологией $\tau$. Пусть $X$ - подмножество из $(G, \tau)$ всех элементов порядков $\neq 2, f$-отображение на $X$, заданное правилом $x \mapsto-x$. Применим $\mathrm{k}$ локальной левотопологическон группе $X$ и однородному автоморфизму $f$ на $X$ 2-го порядка теорему 2. Получим разбиение $X$ на счетное число подмножеств. Каждое из этих подмножеств плотно в $\left(G, \tau^{\prime}\right)$ для любой недискретной групповой топологии $\tau^{\prime}$ на $G$. Для этого достаточно доказать, что каждая окрестностъ нуля в топологии $\tau^{\prime}$ нетривиально пересекает каждую окрестность нуля в топологии $\tau$. Допустим противное: для некоторых окрестностей нуля $U, U^{\prime}$ в топологиях $\tau, \tau^{\prime}$ соответственно $U \cap U^{\prime}=\{0\}$. Выберем окрестности нуля $V, V^{\prime}$ в топологиях $\tau, \tau^{\prime}$ такие, что $V-V \underline{U}, V^{\prime}-V^{\prime} \subseteq U^{\prime}$. Тогда для любых различных $a, b \in V^{\prime}(a+V) \cap(b+V)=\emptyset$ (если бы это было не так, то имели бы $\left.\left(V^{\prime}-V^{\prime}\right) \cap(V-V) \neq\{0\}\right)$. Следовательно, $\tau$ не предкомпактна.

Bonpoc 3. Существует ли бесконечная (счетная) группа с конечным числом элементов порядка 2, не являющаяся абсолютно $\aleph_{0}$-разложимой ?

1. Comfort W. W., van Mill J. Groups with only resolvable group topologies // Proc. Amer. Math. Soc. - 1994. - 120, № 3. - P. $687-696$.

2. Малыхин В. Н. Экстремалын песвязные и близкие к ним группы // Докл. АН СССР. - 1975. $-220, N^{2} 1 .-$ C. $27-30$.

3. Протасов Н. В. Абсолюпно разложимые группы // Укр. мат. журн. - 1996. - 48, № 3. C. $383-392$.

4. Протасов И. В. Разбиения прямых произведений групл // Там же. - 1997. - 49, № 10. C. $1385-1395$.

5. Протасов И. В. Абсолютная разложимость группы рациональных чисел // Там же. 1996. - 48. № 12. - C. 1953 - 1956.

6. Зеленкк Е. Г. Копечные группы в $\beta \mathbb{N}$ тривиальны. - Киев, 1996. - 12 с. - (Препринт / НАН Украины. ЙІ-т математики; № 96.3).

Получено 05.11 .96 